\title{
You said that?
}

Joon Son Chung ${ }^{\star}$

http://www.robots.ox.ac.uk/ joon

Amir Jamaludin ${ }^{\star}$

http://www.robots.ox.ac.uk/ amirj

Andrew Zisserman

http://www.robots.ox.ac.uk/ az
Visual Geometry Group

Department of Engineering Science

University of Oxford

Oxford, UK

\begin{abstract}
We present a method for generating a video of a talking face. The method takes as inputs: (i) still images of the target face, and (ii) an audio speech segment; and outputs a video of the target face lip synched with the audio. The method runs in real time and is applicable to faces and audio not seen at training time.

To achieve this we propose an encoder-decoder CNN model that uses a joint embedding of the face and audio to generate synthesised talking face video frames. The model is trained on tens of hours of unlabelled videos.

We also show results of re-dubbing videos using speech from a different person.
\end{abstract}

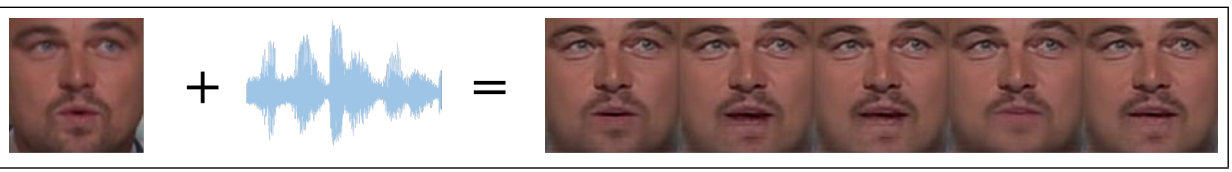

Figure 1: The Speech2Vid model generates a video of a talking face, given still images of the person and a speech segment. The model takes an image of the target face and an audio segment, and outputs a video of the target face lip synched with the audio. Note that the target face need not be in the training dataset i.e. the Speech2Vid is applicable to unseen images and speech.

\section{Introduction}

There has been much work recently in the area of transforming one modality to another.

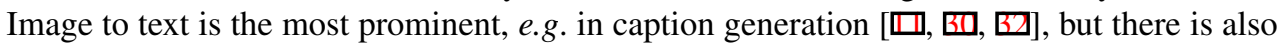
video to sound e.g. [ $[\mathbb{U}$ ], text to image [四], or in fact a mixture of different mediums e.g. video and audio to text [G]. This paper considers the case of audio to video.

We propose a method to generate videos of a talking face using only an audio speech segment and a face image of the target identity (audio and image to video). The speech segment need not be spoken originally by the target person (see Figure 1). We dub the approach Speech2Vid. Our method differs from previous approaches for this task (see related work below) in that instead of learning phoneme to viseme mappings, we learn the correspondences 
between raw audio and video data directly. By focusing on the speech portion of audio and tight facial regions of speakers in videos, the Speech2Vid model is able to produce naturallooking videos of a talking face at test time even when using an image and audio outside of the training dataset.

The key idea of the approach is to learn a joint embedding of the target face and speech segment that can be used to generate a frame of that face saying (lip synched with) the speech segment. Thus the inputs are still images of the face (that provides the identity, but is not speaking the target segment) and the target speech segment; and the generated output is the target face speaking the segment. The Speech2Vid model can be learnt from unlabelled videos, as shown in Figure 7.

In the following, we first describe the automatic pipeline to prepare the video dataset used to train the generation network (Section 2). The architecture and training of the Speech2Vid model is given in Section 3. Finally, Section 4, assesses variations on the architecture, including using multiple images of the identity as input, and shows an application of the model to re-dubbing videos by visually blending the generated face into the source video frame.

\subsection{Related Work}

There are various works that proposed methods to generate or synthesise videos of talking heads from either audio or text sources. Fan et al. [0] introduced a method to restitch the lower half of the face via a bi-directional LSTM to re-dub a target video from a different audio source. The LSTM selects a target mouth region from a dictionary of saved target frames, rather than generating the image, so it requires a sizeable amount of video frames of the unique target identity to choose from. Similarly, Charles et al. [四] uses phonetic labels to select frames from a dictionary of mouth images. Wan et al. [ $[$ ] proposed a method to synthesise a talking head via an active appearance model with the ability to control the emotion of the talking avatar, but they are constrained to the unique model trained by the system. Garrido et al. [0] synthesises talking faces on target speakers by transferring the mouth shapes from the video of the dubber to the target video, but this method requires the video footage of the dubber's mouth saying the speech segment, whereas our method learns the relationship between the sound and the mouth shapes.

Our training approach is based on unsupervised learning, in our case from tens of hours of people talking. One of the earliest example of unsupervised learning of the representation of data using neural networks is the autoencoder by Hinton and Salakhutdinov [ $\mathrm{\theta}]$. Further improvements in representation learning, for example the variational auto-encoders by Kingma et al. [ $\square]$, opens up the possibility of generating images using neural networks. Moving forward, current research shows adversarial training proposed by [छ] works well for generating natural-looking images; conditional generative models [ $[\mathbf{⿴ 囗 十}]$ are able to generate images based on auxilary information such as a class label. Our Speech2Vid model is closest in spirit to the image-to-image model by Isola et al. [四] in that we generate an output that closely resembles the input, but in our case we have both audio and image data as inputs.

\section{Dataset}

This section describes our multi-stage strategy to prepare a large-scale dataset to train the generation network. We obtain tens of hours of visual face sequences aligned with spoken audio. 
The principal stages are: (i) detect and track all face appearances in the video; (ii) determine who is speaking in the video; and (iii) align the detected face image to the canonical face. The pipeline is summarised in Figure 2, and the details are discussed in the following paragraphs.

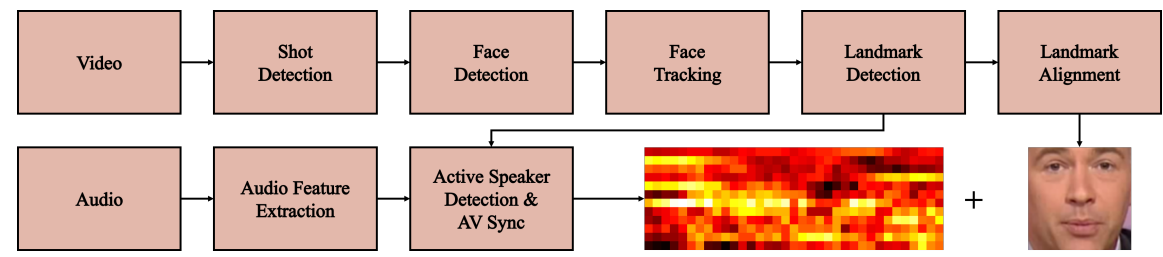

Figure 2: Data preparation pipeline.

Video description. We train the Speech2Vid model on videos from the VoxCeleb [四] and LRW [ $⿴ 囗$ ] datasets. These datasets consist of celebrity interviews and broadcast news, which provide ideal training data for this task, given that a large proportion of the face tracks are front-facing, and of high quality. Moreover, the words are generally clearly spoken without too much background noise, and hence provide an easier learning environment for the network.

Face tracking. The face tracking pipeline is based on [曰]. First, the shot boundaries are determined by comparing colour histograms $[\square]$ to find the within-shot frames for which tracking is to be run. The HOG-based DLIB face detector []] is used to detect face appearances on every frame of the video. The face detections are grouped into face tracks using a KLT detector [ष]]. Facial landmarks are extracted using the regression-tree based method of [四].

Active speaker detection and AV synchronisation. SyncNet [ $[$ ] provides a joint embedding of the audio and visual face sequences in a video, which can be used to determine who is speaking in a multi-speaker video scene. Moreover, the same method is used to correct the lip-sync error in the broadcast video, which can be crucial for precisely locating the corresponding mouth image for the audio sample.
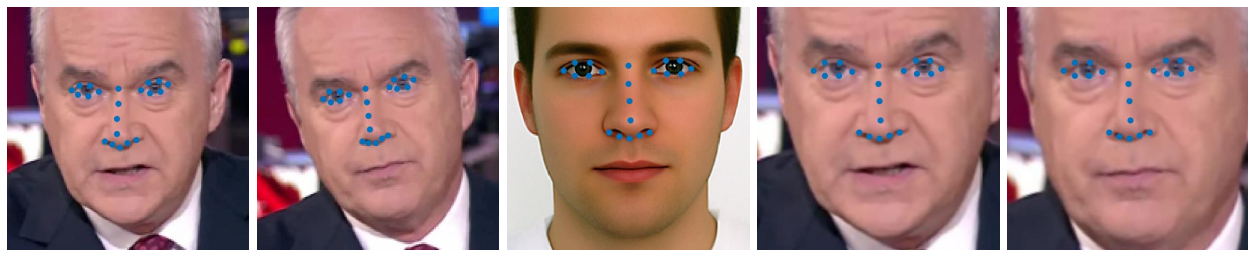

Figure 3: Left pair: Face images before registration; Middle: Canonical face; Right pair: Face images after registration with the canonical face.

Spatial registration. In order to establish spatial correspondance between the input face (that provides the identity to the encoder) and the output face (from the decoder) in training from the ground truth frames, we register the facial landmarks between the two images. This 
is done by performing a similarity transformation (scale, rotation and translation) between the faces and an exemplar face with canonical position (Figure 3 middle). Only the landmarks on the eyes and the nose, not the mouth, are used to align the face image, as the mouth movements contain the information that we wish to capture.

Data statistics. The train-validation split is given in the VoxCeleb and LRW datasets. For every valid face track, we extract every 5 th frame and the corresponding audio as samples for training and validation. Statistics on the dataset is given in Table 1.

\begin{tabular}{|l|r|r|}
\hline Set & \# Hours & \# Samples \\
\hline \hline Train & 37.7 & 678,389 \\
\hline Val & 0.5 & 9,287 \\
\hline
\end{tabular}

Table 1: Dataset statistics

\section{The Speech2Vid Model}

Our main goal at test time is to generate a video of a talking face given two inputs: (i) an audio segment, and (ii) still images of the target identity (frontal headshot). The Speech2Vid model (summarised in Figure 4 at the block level), consists of four main components: an audio encoder, an identity image encoder, a talking face image decoder, and a deblurring module. For a given input sample, the model generates one frame of image output that best represents the audio sample at a specific time step. The model generates the video on a frame-by-frame basis by sliding a 0.35 -second window over the audio sequence.

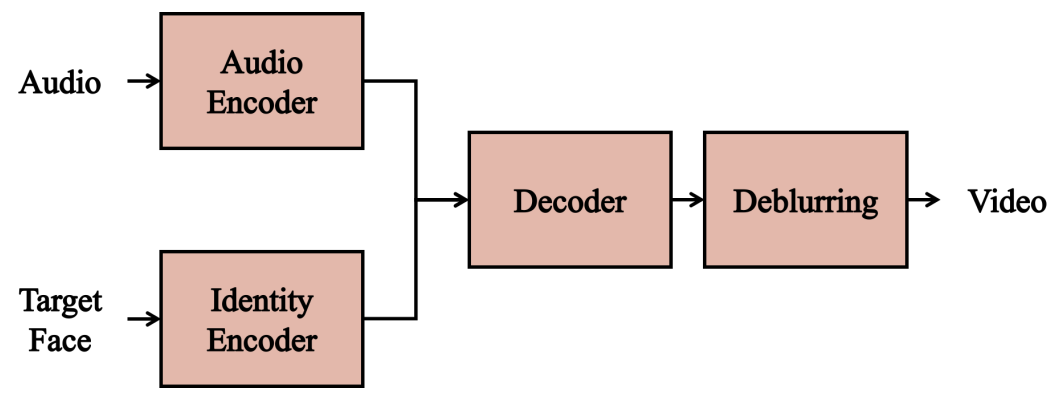

Figure 4: The overall Speech2Vid model is a combination of two encoders taking in two different streams of data, audio and image, a decoder that generates images corresponding to the audio, and a CNN deblurring module that refine the output frames.

\subsection{Input Representations}

This section describes the input representations for the audio and identity. These inputs are fed into separate modules in the network in the forms of 0.35 -second audio and a still image of the target identity.

Audio. The input to the audio encoder are Mel-frequency cepstral coefficients (MFCC) values extracted from the raw audio data. The MFCC values are made up of individual 
coefficient each representing a specific frequency band of the audio short-term power on a non-linear mel scale of frequency; 13 coefficients are calculated per sample but only the last 12 are used in our case. Each sample fed into the audio encoder is made up of 0.35-second input audio data with a sampling rate of $100 \mathrm{~Hz}$ resulting in 35 time steps. Each encoded sample can be viewed as a $12 \times 35$ heatmap where each column represents MFCC features at each time step (see Figure 5).
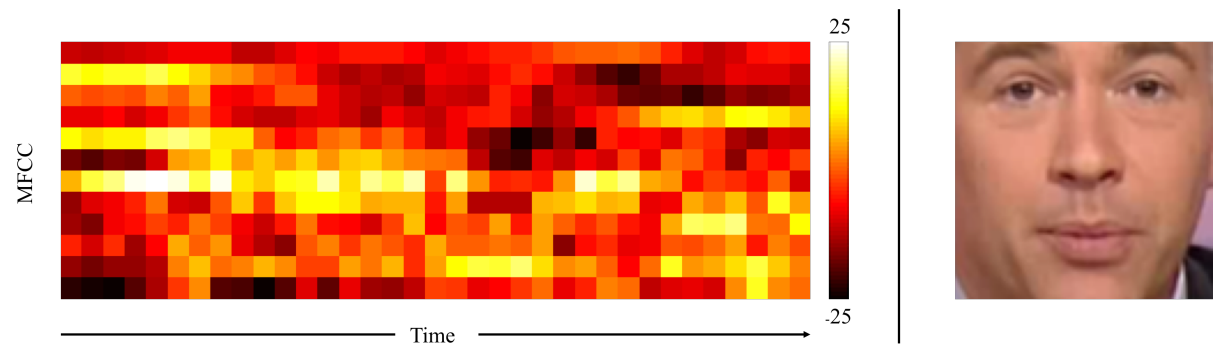

Figure 5: Inputs to the Vid2Speech model. Left: MFCC heatmap for the 0.35-second time period. The 12 rows in the matrix represent the power of the audio at different frequencies. Right: Still image of the speaker.

Identity. The input to the identitiy encoder is a single still image with dimensions $112 \times$ $112 \times 3$. In Section 4.2, we also experiment with having multiple still images as the input to the identity encoder instead of one, which significantly improves the output video quality.

\subsection{The Architecture}

The Speech2Vid architecture is given in Figure 6. We describe the three modules (audio encoder, the identity encoder, and the image decoder) in the following paragraphs. Note, these three modules are trained together. The deblurring module (described below in Section 3.3) is trained separately.

Audio encoder. We use a convolutional neural network originally designed for image recog-

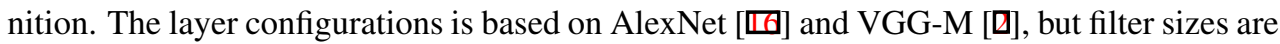
adapted for the unusual input dimensions. This is similar to the configuration used to learn audio embedding in [四].

Identity encoder. Ideally, the identity vector produced by the encoder should have features unique for facial recognition and as such we use a VGG-M network pre-trained on the VGG Face dataset $[\square]$. The dataset includes $2.6 \mathrm{M}$ images of $2.6 \mathrm{~K}$ unique identities. Only the weights of the convolutional layers are used in the encoder, while the weights of the fullyconnected layers are reinitialized.

Image decoder. The decoder takes as input the concatenated feature vectors of the FC7 layers of the audio and identity encoders (both 256-dimensional). The features vector is gradually upsampled, layer-by-layer via transposed convolutions. See details in Figure 6. The network features two skip connections to help preserve the defining features of the target identity - this is done by concatenating the encoder activations with the decoder activations 


\section{Audio Encoder}

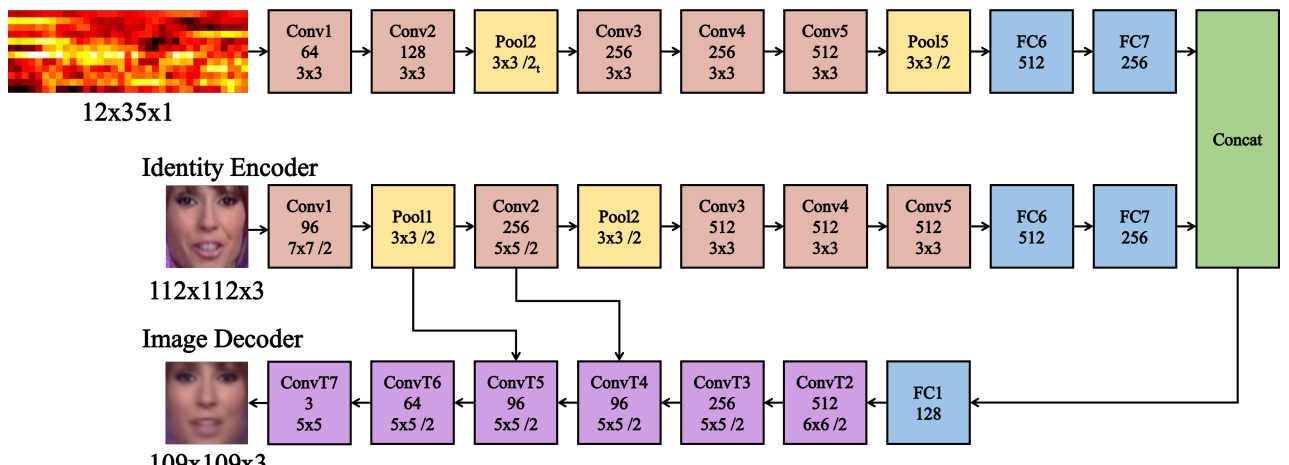

Figure 6: The three modules in the Speech2Vid model. From top to bottom: (i) audio encoder, (ii) identity encoder with a single still image input, and (iii) image decoder. $/ 2$ refers to the stride of each kernel in a specific layer which is normally of equal stride in both spatial dimensions except for the Pool2 layer in which we use stride 2 in the timestep dimension (denoted by $/ 2_{t}$ ). The network includes two skip connections between the identity encoder and the image decoder.

(as suggested in [四]) at the locations shown in the network diagram.

Loss function. An $L_{1}$ loss is used (Equation 1), rather than $L_{2}$ that is more commonly used for image generation and in auto-encoders, as $L_{1}$ tends to encourage less blurring [四].

$$
\mathcal{L}=\sum_{n=1}^{N}\left\|\hat{y}_{n}-y_{n}\right\|
$$

Training protocol. The network is trained on the video dataset described in Section 2. During training, the ground truth output image of the target identity speaking the audio segment is used as supervision. The image is taken from the middle frame of the video in the 0.35 -second sampling window. The image for the input identity of the speaker is randomly sampled from a different point in time, as shown in Figure 7). When multiple still images are used for the input identity (Section 4.2) we randomly sample multiple images from the same video stream.
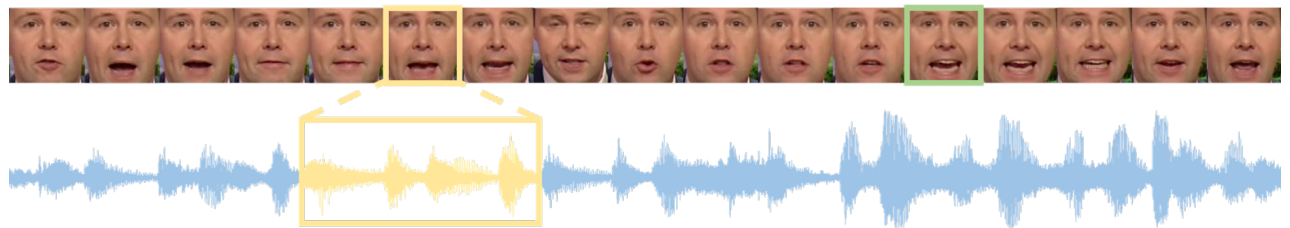

Figure 7: Sampling strategy for identity images during training. Identities are randomly sampled from future frames far from actual audio/output image samples. 
Discussion. The network architecture is based purely on ConvNets, as opposed to the recurrent architectures often used for tasks relating to time sequences. The rationale is that the mouth shape of the speaker does not depend on anything other than the phoneme that is being said at the exact moment, and the long term context is unimportant. We find that the 0.35 -second window is more than enough to capture this information. At test time, the video is generated frame-by-frame by sliding a temporal window across the entire audio segment while using the same single identity image.

Implementation details. Our implementation is based on the MATLAB toolbox MatConvNet [ $\mathrm{Z}]$ and trained on a NVIDIA Titan X GPU with 12GB memory. The network is trained with batch normalisation and a fixed learning rate of $10^{-5}$ using stochastic gradient descent with momentum. The training was stopped after 20 epochs, or when the performance on the validation set stops improving, whichever is sooner.

At test time, the network (including the deblurring layers) runs faster than twice real-time on a GPU. This can be further accelerated by pre-computing and saving the features from the identity encoder module, rather than running this for every frame. In the case of redubbing video, the the output video is generated at the same frame rate as the original video.

\subsection{Deblurring module}

CNNs trained to generate images with $L_{1}$ and $L_{2}$ losses tend to produce blurry images [ $\square$, [3]. To mitigate this problem, we train a separate deblurring CNN to sharpen the images produced by the Speech2Vid model. The model is inspired by VDSR []], which uses a residual connection between the input and output, so that the network only has to learn the image difference. Our implementation has 10 convolutional and ReLU layers, and the layer configuration is shown in Figure 8.

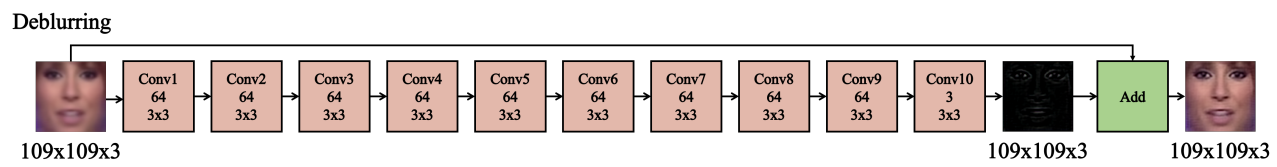

Figure 8: Deblurring CNN module

We train the network on artificially blurred face images (Figure 9), as opposed to training the network end-to-end together with the generator network. This is because the alignments between the input image, the target (ground truth) image and the generated image are not perfect even after the spatial registration (of Section 2), and thus avoid the deblurring network having to learn the residual coming from the misalignment.

The images that we ask the CNN to deblur are relatively homogeneous in content (they are all face images), and we find that the $\mathrm{CNN}$ performs very well in sharpening the images under this constraint.

\section{Experiments}

\section{The results are best seen in video format. Please refer to the online examples.}

Figure 10 shows a visualization of the output of the model (the frames of the two segments highlighted in the captions "major" and "based on"). Note, the movement of the 

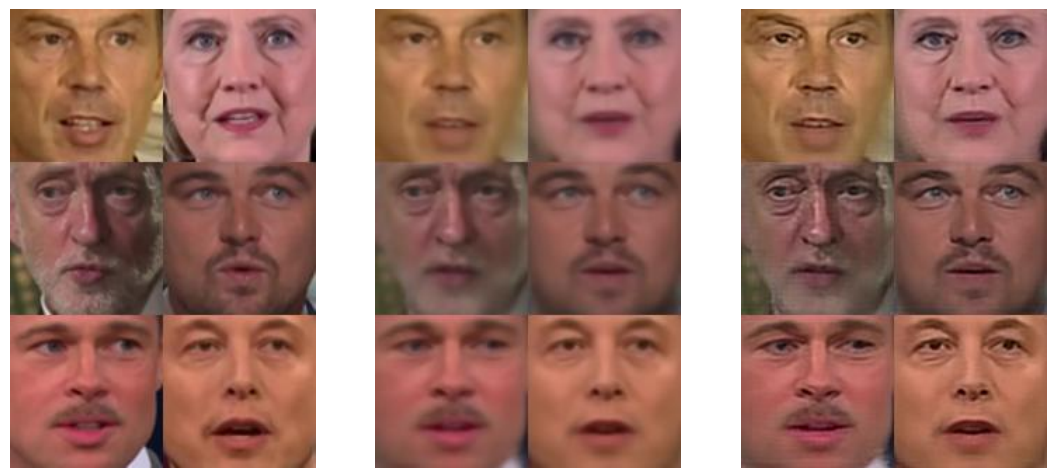

Figure 9: Deblurring CNN input and output. Left: Original face image (ground truth); Middle: Input to the deblurring CNN; Right: Restored face image using the deblurring CNN.
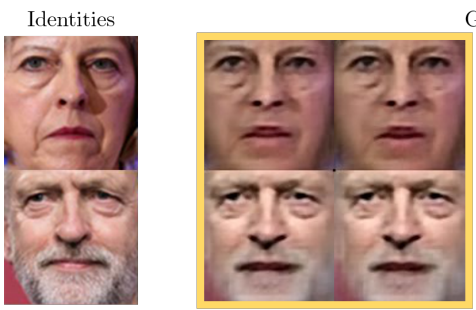

Generated frames

This is the first major study of its kind but, presumably its based on ..

Figure 10: Top row: Identity 1 and the corresponding generated frames; Middle row: Identity 2 and the corresponding generated frames; Bottom row: Captions of the audio segment. Best seen in video form.

mouths of the two examples reflect the sound of each word not unlike phoneme-to-viseme correspondences.

\subsection{Preserving Identity with Skip Connections}

Figure 11 shows a set of generated faces and various target identities (original stills). We observe that the skip connections are crucial to carry facial features from the input image to the generated output - without these, the generated images lose defining facial features of target identities, as shown in the middle column. The skip connections at earlier layers (e.g. after conv1) were not used as it encouraged the output image to be too similar to the still input, often restricting the mouth shapes that we want to animate.

\subsection{Preserving Identity with Multiple Still Images}

Instead of a single image specifying the unique identity, five distinct images are concatenated channel-wise resulting in an input dimension of $112 \times 112 \times 15$, and the convl layer of the identity encoder are modified to ingest inputs of these dimensions. In training these examples 


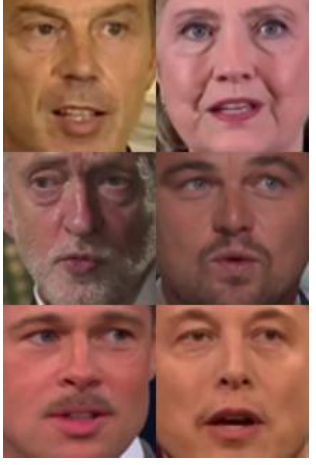

(a)

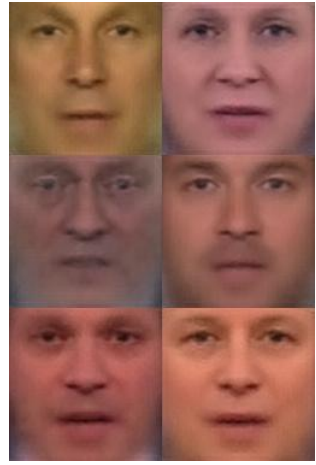

(b)

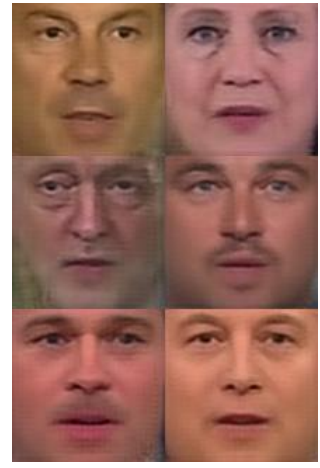

(c)

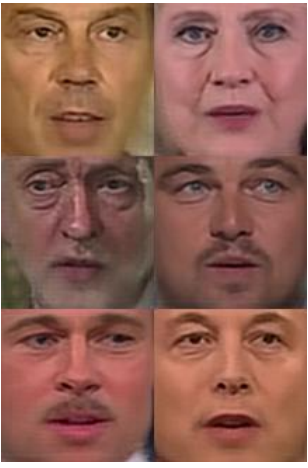

(d)

Figure 11: (a) Original still image to animate (input to the identity encoder); (b) Output frames without skip connection; (c) Output frames with skip connection and one input image; (d) Output frames with skip connection and five input images. It is clear that the skip connection helps to carry facial features over from the identity image to the generated video frames.

are sampled with a similar strategy to that of Figure 7, but multiple 'identity' images are sampled, instead of just one.

As can be seen in Figure 11, having multiple image examples for the unique identity enhances the quality of the generated faces. There are two reasons for this: first, with multiple example images as input, it is likely that the network now has access to images of the person showing the mouth open as well as closed. Thus, it has to hallucinate less in generation as, in principle, more can be sourced directly from the input images; Second, although the faces are aligned prior to the identity encoder, there are minor variations in the movement of the face other than the lips that are not relevant to the speech, from blinking and microexpression. The impact of these minor variations when extracting unique identity features is reduced by having multiple still images of the same person.

\subsection{Application: Lip Transplant/Re-dubbing Videos}

The Speech2Vid model can be applied to visually re-dub a source video with a different segment of spoken audio. The key stages are as follows: (i) obtain still images from the source video for identity; (ii) generate the face video for the given audio and identity using the Speech2Vid model; (iii) re-align the landmarks of the generated video to the source video frames, and (iv) visually blend the aligned face with the source video frame.

Alignment. Facial landmarks in the target video is determined using the method of [ $\square]$ ]. A similarity transformation is used to align the generated face with the original face in the target image. Figure 12 (right) shows the generated face in alignment with the original face.

Poisson editing. The Poisson blending algorithm [四] blends two images together by matching gradients with boundary conditions. We use this technique to match the generated face with the source video frame, as shown in Figure 12. This can be used to blend the face from the same, or different identity to the source video frame. 


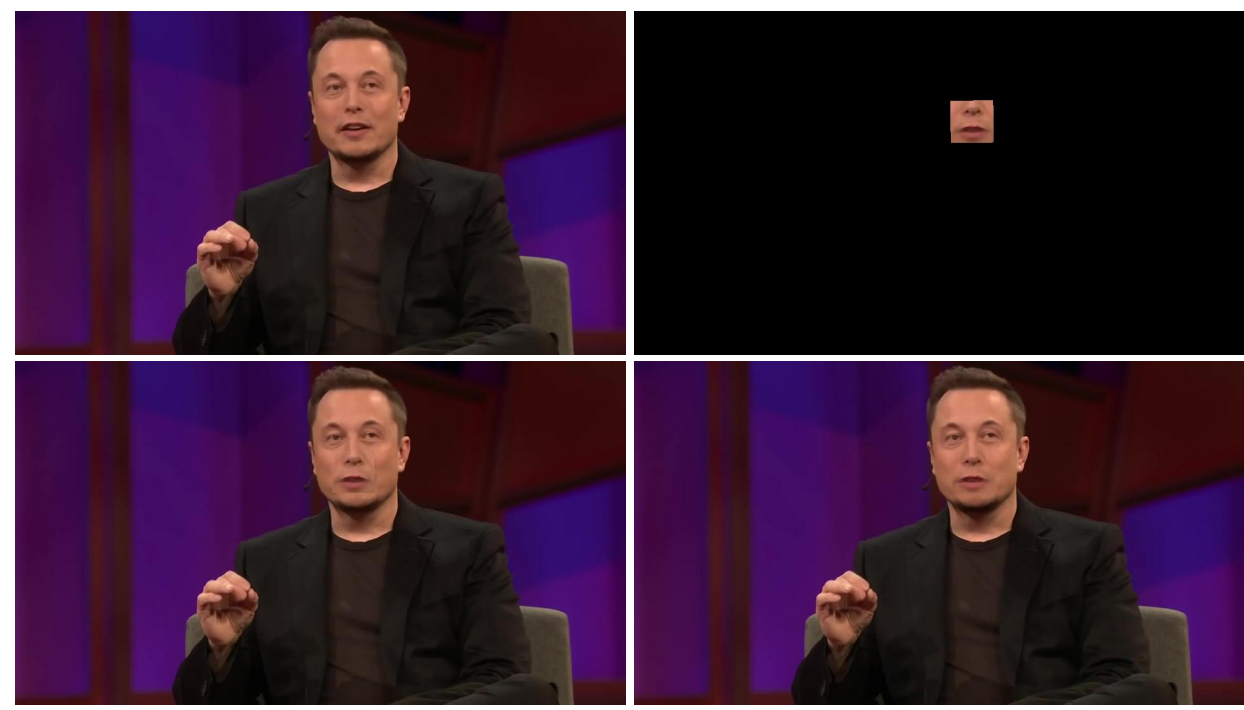

Figure 12: Top left: Original still image; Top right: Generated mouth region, aligned with the original (target) face; Bottom left: Generated mouth region, superimposed on the original face. Bottom right: Generated mouth region, blended with the original face.

Discussion. This method can be used to blend the generated face as a whole, or to match only the lower half of the face. We qualitatively find that we strike the best balance between image naturalness and movement naturalness by only blending the lower half of the face, from just below the eyes.

\section{Summary and extensions}

We have demonstrated that the Speech2Vid model is able to generate videos of any identity speaking from any source of input audio. This work shows that there is promise in generating video data straight from an audio source. We have also shown that re-dubbing videos from a different audio source (independent of the original speaker) is possible.

One clear extension is to add a quantitative performance measure of our models. This is not a straightforward task as there is no definitive performance measure of generative models for a specific domain. In natural image generation, Salimans et al. [ $\square]$ ] proposed a scoring system dependent on an image's softmax output when fed into a network trained on a classification task e.g. the inception network trained on ImageNet [ $\mathbb{Z}]$. One possible option is to have a lip-specific inception score using networks trained on a lip-specific task [ $[$ ] .

Moving forward, this model can be applied to computer facial animation relying only on audio.

Acknowledgements. Funding for this research is provided by the EPSRC Programme Grant Seebibyte EP/M013774/1. Amir Jamaludin is funded by the RCUK CDT in Healthcare Innovation EP/G036861/1. We would like to thank Aravindh Mahendran for helpful discussions. 


\section{References}

[1] Charles, J., Magee, D., Hogg, D.: Virtual immortality: Reanimating characters from tv shows. In: Computer Vision-ECCV 2016 Workshops. pp. 879-886. Springer (2016)

[2] Chatfield, K., Simonyan, K., Vedaldi, A., Zisserman, A.: Return of the devil in the details: Delving deep into convolutional nets. In: Proc. BMVC. (2014)

[3] Chung, J.S., Senior, A., Vinyals, O., Zisserman, A.: Lip reading sentences in the wild. In: Proc. CVPR (2017)

[4] Chung, J.S., Zisserman, A.: Lip reading in the wild. In: Proc. ACCV (2016)

[5] Chung, J.S., Zisserman, A.: Out of time: automated lip sync in the wild. In: Workshop on Multiview Lip-reading, ACCV (2016)

[6] Fan, B., Wang, L., Soong, F.K., Xie, L.: Photo-real talking head with deep bidirectional lstm. In: Acoustics, Speech and Signal Processing (ICASSP), 2015 IEEE International Conference on. pp. 4884-4888. IEEE (2015)

[7] Garrido, P., Valgaerts, L., Sarmadi, H., Steiner, I., Varanasi, K., Pérez, P., Theobalt, C.: Vdub: Modifying face video of actors for plausible visual alignment to a dubbed audio track. In: Computer Graphics Forum. vol. 34, pp. 193-204. Wiley Online Library (2015)

[8] Goodfellow, I.J., Pouget-Abadie, J., Mirza, M., Xu, B., Warde-Farley, D., Ozair, S., Courville, A.C., Bengio, Y.: Generative adversarial nets. In: Advances in Neural Information Processing Systems 27: Annual Conference on Neural Information Processing Systems 2014, December 8-13 2014, Montreal, Quebec, Canada. pp. 2672-2680 (2014), http: / / papers .nips . cc/ paper/5423-generative-adversarial-nets

[9] Hinton, G.E., Salakhutdinov, R.R.: Reducing the dimensionality of data with neural networks. Science 313(5786), 504-507 (Jul 2006), http://www.ncbi.nlm.nih.gov/sites/ entrez?db=pubmed\&uid=16873662\&cmd=showdetailview\&indexed=google

[10] Isola, P., Zhu, J.Y., Zhou, T., Efros, A.A.: Image-to-image translation with conditional adversarial networks. arxiv (2016)

[11] Karpathy, A., Fei-Fei, L.: Deep visual-semantic alignments for generating image descriptions. In: Proc. CVPR. pp. 3128-3137 (2015)

[12] Kazemi, V., Sullivan, J.: One millisecond face alignment with an ensemble of regression trees. In: Proceedings of the IEEE Conference on Computer Vision and Pattern Recognition. pp. 18671874 (2014)

[13] Kim, J., Lee, J.K., Lee, K.M.: Accurate image super-resolution using very deep convolutional networks. In: Proc. CVPR (June 2016)

[14] King, D.E.: Dlib-ml: A machine learning toolkit. The Journal of Machine Learning Research 10, 1755-1758 (2009)

[15] Kingma, D.P., Welling, M.: Auto-encoding variational bayes. CoRR abs/1312.6114 (2013), http://arxiv.org/abs/1312.6114

[16] Krizhevsky, A., Sutskever, I., Hinton, G.E.: ImageNet classification with deep convolutional neural networks. In: NIPS. pp. 1106-1114 (2012) 
[17] Lienhart, R.: Reliable transition detection in videos: A survey and practitioner's guide. International Journal of Image and Graphics (Aug 2001)

[18] Lucas, B.D., Kanade, T.: An iterative image registration technique with an application to stereo vision. In: Proc. of the 7th International Joint Conference on Artificial Intelligence. pp. 674-679 (1981), citeseer.nj.nec.com/lucas81optical.html

[19] Nagrani, A., Chung, J.S., Zisserman, A.: Voxceleb: a large-scale speaker identification dataset. In: INTERSPEECH (2017)

[20] van den Oord, A., Kalchbrenner, N., Espeholt, L., Vinyals, O., Graves, A., et al.: Conditional image generation with pixelcnn decoders. In: Advances in Neural Information Processing Systems. pp. 4790-4798 (2016)

[21] Owens, A., Isola, P., McDermott, J.H., Torralba, A., Adelson, E.H., Freeman, W.T.: Visually indicated sounds. In: CVPR. pp. 2405-2413. IEEE Computer Society (2016), http : / / db lp. uni-trier.de/db/conf/cvpr/cvpr2016.html\#OwensIMTAF1 6

[22] Parkhi, O.M., Vedaldi, A., Zisserman, A.: Deep face recognition. In: Proc. BMVC. (2015)

[23] Pathak, D., Krahenbuhl, P., Donahue, J., Darrell, T., Efros, A.A.: Context encoders: Feature learning by inpainting. In: Proc. CVPR. pp. 2536-2544 (2016)

[24] Perez, P., Gangnet, M., Blake, A.: Poisson image editing. ACM Transactions on Graphics 22(3), 313-318 (2003)

[25] Reed, S.E., Akata, Z., Yan, X., Logeswaran, L., Schiele, B., Lee, H.: Generative adversarial text to image synthesis. In: Balcan, M.F., Weinberger, K.Q. (eds.) ICML. JMLR Workshop and Conference Proceedings, vol. 48, pp. 1060-1069. JMLR.org (2016), http: / / dblp . uni-trier.de/db/conf/icml/icml2016.html\#ReedAYLSL1 6

[26] Ronneberger, O., Fischer, P., Brox, T.: U-net: Convolutional networks for biomedical image segmentation. In: International Conference on Medical Image Computing and Computer-Assisted Intervention. pp. 234-241. Springer (2015)

[27] Salimans, T., Goodfellow, I.J., Zaremba, W., Cheung, V., Radford, A., Chen, X.: Improved techniques for training gans. CoRR abs/1606.03498 (2016), http://arxiv . org/abs/1606. 03498

[28] Szegedy, C., Liu, W., Jia, Y., Sermanet, P., Reed, S., Anguelov, D., Erhan, D., Vanhoucke, V., Rabinovich, A.: Going deeper with convolutions. In: Proc. CVPR (2015)

[29] Vedaldi, A., Lenc, K.: Matconvnet: Convolutional neural networks for matlab. In: Proc. ACMM (2015)

[30] Vinyals, O., Toshev, A., Bengio, S., Erhan, D.: Show and tell: A neural image caption generator. In: Proceedings of the IEEE Conference on Computer Vision and Pattern Recognition. pp. 31563164 (2015)

[31] Wan, V., Anderson, R., Blokland, A., Braunschweiler, N., Chen, L., Kolluru, B., Latorre, J., Maia, R., Stenger, B., Yanagisawa, K., et al.: Photo-realistic expressive text to talking head synthesis. In: INTERSPEECH. pp. 2667-2669 (2013)

[32] Xu, K., Ba, J., Kiros, R., Courville, A., Salakhutdinov, R., Zemel, R., Bengio, Y.: Show, attend and tell: Neural image caption generation with visual attention. arXiv preprint arXiv: 1502.03044 (2015)

[33] Zhang, R., Isola, P., Efros, A.A.: Colorful image colorization. In: Proc. ECCV. pp. 649-666. Springer (2016) 